\title{
Anciens et nouveaux terroirs d'étangs en Lorraine
}

old and new ponds landscapes in Lorraine

Alte und neue Weiherlandschaften in Lothringen

Denis Mathis and Anne Mathis

\section{CpenEdition}

\section{Journals}

Electronic version

URL: http://journals.openedition.org/rge/5712

ISSN: 2108-6478

\section{Publisher}

Association des géographes de l'Est

Printed version

Date of publication: 22 April 2016

ISSN: 0035-3213

\section{Electronic reference}

Denis Mathis and Anne Mathis, "Anciens et nouveaux terroirs d'étangs en Lorraine », Revue Géographique de l'Est [Online], vol.56 / n¹-2 | 2016, Online since 29 August 2016, connection on 01 May 2019. URL : http://journals.openedition.org/rge/5712

This text was automatically generated on 1 May 2019.

Tous droits réservés 


\title{
Anciens et nouveaux terroirs d'étangs en Lorraine
}

\author{
Old and new ponds landscapes in Lorraine \\ Alte und neue Weiherlandschaften in Lothringen
}

Denis Mathis and Anne Mathis

1 Aujourd'hui et de longue date, la Lorraine est une importante région piscicole en France ${ }^{1}$. Pourtant, ce savoir-faire reste finalement assez méconnu et peu mis en valeur à échelle régionale et nationale, notamment par rapport aux autres vieux « pays d'étangs » tels que les Dombes, la Brenne ou encore la Sologne. Qui en France connaît les grands étangs de Lorraine et ce bien que la tradition locale rapporte que les carpes de l'étang de Lindre aient été servies à la table royale ou encore aient alimenté les bassins de Fontainebleau ? Cette méconnaissance par rapport aux grandes régions d'étangs est la conséquence de constructions identitaires tardives des «pays d'étangs » lorrains, mais aussi de la variété et de la multiplicité de ces derniers. La Lorraine n'abrite pas un, mais plusieurs pays d'étangs : Pays de Bitche, Woëvre, Pays de l'Albe et des Lacs, Pays des Étangs sont des petits territoires aux multiples identités. Les assolements piscicoles étaient complémentaires et ces territoires étaient modelés par une gestion agricole et/ou sylvicole, voire proto-industrielle. Le particularisme des eaux stagnantes lorraines n'était souvent qu'une facette de la structure paysagère et identitaire du territoire. Cette activité piscicole secondaire est propre à quelques grands propriétaires fonciers pesant sur des communautés rurales, d'où l'absence de mécanisme de production identitaire et socioculturelle des pays d'étangs qu'ont pu souligner G. Bédoucha (2011) pour la Brenne ou V. Manceron (2004, 2006a et 2006b) pour les Dombes. En Lorraine, la notion de «pays d'étangs » s'est construite artificiellement, particulièrement dans le cadre du « Pays des Étangs » en Moselle. La construction des identités « des territoires d'eau » est récente et désormais inscrite au sein de projets territoriaux (Chiffre et Mathis, 2013 ; 2014 ; Mathis, 2014).

2 Le patrimoine lentique lorrain concerne de très grands étangs : Madine, Stock ou Lindre, nés de la coalescence de plusieurs étangs que l'on pourrait presque qualifier de «lacs- 
étangs » en adaptant la terminologie de P. Papon et L. Touchart (2003) et en l'adaptant à la référence plus modeste des étangs de la Lorraine. Il s'agit le plus souvent de zones humides multifonctionnelles où l'activité piscicole apparaît désormais plus comme une forme archaïque dont la permanence permet d'assurer un marketing territorial, comme à l'étang de Lachaussée ou au Domaine du Lindre par les activités événementielles des "grandes pêches ». Les enjeux actuels dans la protection, la préservation, la gestion, l'aménagement écologique ou anthropique expriment les nouvelles approches liées à la protection de ces espaces qui se heurtent finalement aux usages traditionnels des zones d'étangs. Les conflits liés aux étangs ont toujours été relativement fréquents entre les grands propriétaires et les communautés villageoises. Aujourd'hui, c'est à l'heure où la filière lorraine tente de s'organiser en lien avec les instances administratives, que la question de la gestion de l'eau s'inscrit dans le cadre du pôle de compétitivité « hydréos » et que se définissent les politiques de protections et de valorisation territoriale ; un bilan apparaît alors nécessaire concernant le patrimoine lentique lorrain. Cet inventaire doit tout autant présenter les origines des étangs, les dynamiques à la fois économiques et environnementales et inscrire celles-ci dans le cadre d'une démarche durable voulue par les acteurs politiques et économiques. La diversité des étangs lorrains est une richesse et un patrimoine à découvrir pour permettre sa préservation. Notre propos s'organisera donc en trois temps. D'une part, sans être exhaustif, présenter l'origine et les usages d'un domaine lentique de près de 7000 ha. Dans un second temps souligner les dynamiques actuelles de production des territoires des anciens terroirs d'étangs et les politiques de protections et de préservations mises en œuvre notamment par le Parc Naturel Régional de Lorraine (PNRL). Enfin, dans le cadre des reconversions des anciennes exploitations de gravières, s'interroger sur la place des nouveaux terroirs d'étangs au sein de l'espace lorrain suivant la logique de production des trames bleues et de l'utilisation rationnelle des masses d'eau des étangs.

\section{I. À l'origine d'un patrimoine lentique : des usages divers}

3 Le patrimoine lentique de la Lorraine est très diversifié. Il occupe les vallées des hautes et basses Vosges, la Vôge, les terrains de la Lorraine sédimentaire, les fonds de vallées sédimentaires et investit les espaces périurbains (fig. 1). Si l'origine de ce patrimoine est liée à l'activité piscicole, cette dernière n'a souvent été que complémentaire et associée à une organisation agricole, proto-industrielle ou industrielle. 
Figure 1. La richesse du patrimoine lentique lorrain.

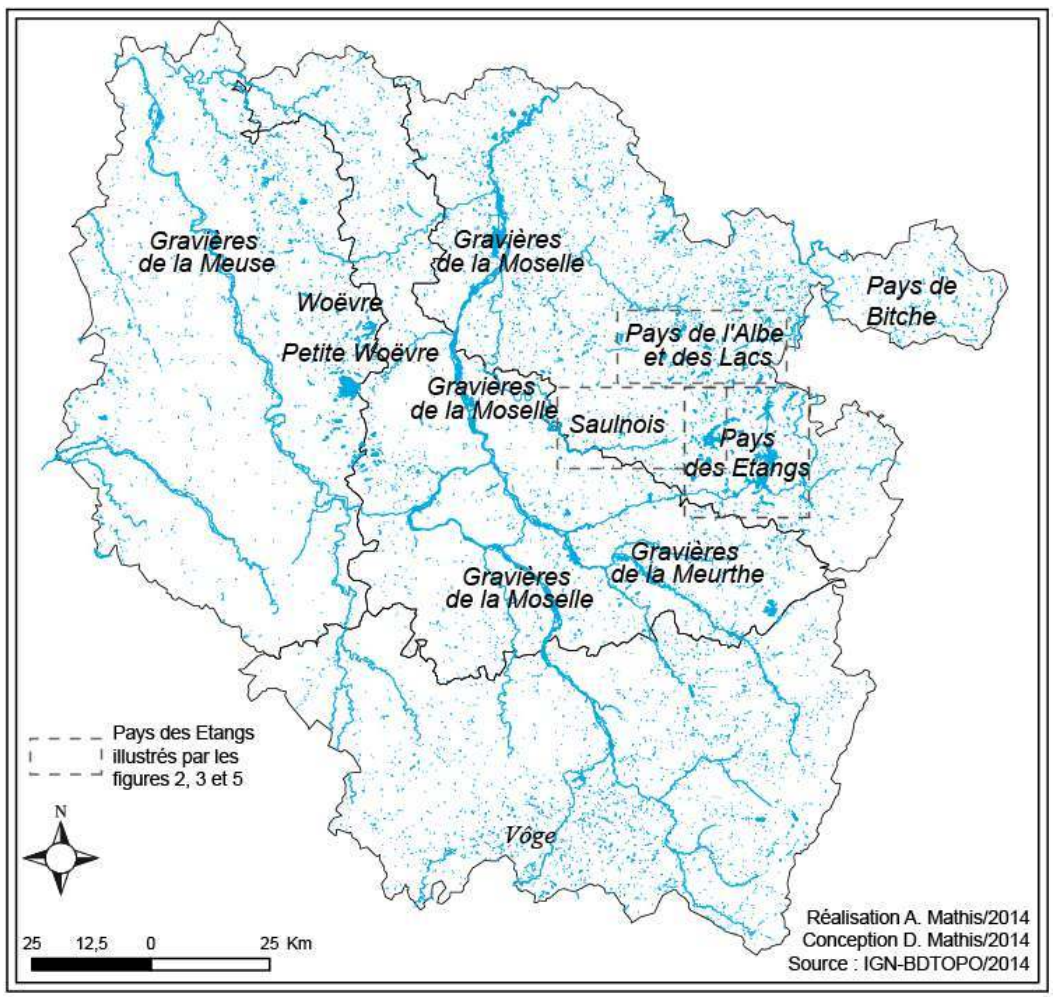

\section{A. L'héritage médiéval de la maîtrise des bassins hydrographiques}

Les étangs lorrains sont des éléments de systèmes agro-piscicoles construits dans des contextes géographiques différents. En effet, ces stagnosystèmes constituent deux modèles de pisciculture, l'un propre à l'espace vosgien (hautes et basses Vosges, Vôge...), l'autre propre à la lorraine sédimentaire du relief de côte où alternent dépressions argilomarneuses, côtes et revers de plateau. C'est dans les grandes dépressions argileuses que se nichent en fond de vallons les étangs et les grands lacs-étangs. Dans ces deux situations, les terroirs d'étangs ont été façonnés pour une mise en valeur piscicole, essentiellement durant le Moyen-Âge. Elle permettait alors de bonifier des espaces jugés médiocres (sols argilo-marneux lourds et difficiles à travailler, zones humides, landes et marais). Digues et chaussées ont progressivement coupé les écoulements de surfaces de petits ruisseaux, élevant le niveau des eaux, profitant parfois de la présence naturelle de tourbières, de mares et de mardelles ${ }^{2}$ pour former des étangs. Ces derniers occupent les fonds de vallées, colonisent parfois l'ensemble d'un ruisseau ou s'organisent en cascade ou en chapelet.

5 Certes ces étangs pouvaient également servir à approvisionner en eau un moulin, mais avec l'essor des marchés urbains, les grands étangs lorrains sont devenus nettement à vocation piscicole. Cette dernière s'est développée et intensifiée avec l'introduction de la carpe vers le XIII siècle (Hoffmann, 1994 ; Benoit, 2002 ; Benarrous et Marinval, 2006). Il est probable que cette nouvelle espèce piscicole, facilement transportable, ait été à l'origine de l'essor de la pisciculture en Lorraine sédimentaire. Elle complète ainsi l'élevage piscicole de la truite établi dans les domaines vosgiens. Il conviendrait également de mettre en parallèle cet essor avec l'état général des cours d'eau et la 
dégradation apparente des pêcheries de rivière. Cette dernière est la conséquence d'une détérioration du milieu hydrographique (atterrissement, étiage, disparition de la ripisylve...) (Mathis D. et A., 2013). Tous ces phénomènes concourent à stimuler la pisciculture d'étangs dont les revenus sont loin d'être négligeables (Mathis, 2009). Cette activité était aux mains des seigneurs, qu'ils soient ecclésiastiques ou laïcs.

Comme pour les régions voisines de Bourgogne et de Champagne (Berthier et alii, 2005), les grands domaines abbatiaux, et notamment cisterciens, ont été des acteurs importants dans l'élaboration de terroirs d'étangs. Installés dans des " déserts », au cœur de l'espace "sauvage », la maîtrise hydrographique apparaît comme un acte fort de construction et d'appropriation du territoire. Ainsi, l'abbaye de Sturzelbronn ${ }^{3}$ dans le Pays de Bitche dispose de 15000 arpents (12000 arpents de bois peu exploitables faute de capacités de flottage et de 3000 arpents de terre, étangs et marais exploités par une vingtaine de granges). Son patrimoine se compose à son apogée de quatorze étangs, dont le plus important était le Grafenweiher'. Ce dernier s'étendait sur près d'un kilomètre de long. Compte tenu de l'importance du domaine lentique et de la faiblesse de la communauté monastique, il est probable que dès les origines les moines aient commercialisé leur production piscicole. Bien que construite dans un « désert ", au cœur du massif forestier des Vosges du Nord, l'abbaye de Sturzelbronn (57) est établie sur l'ancienne voie romaine de Bitche à Wissembourg et dispose ainsi d'un axe pour écouler ses productions. La militarisation de cet espace à partir des $\mathrm{XIII}^{\mathrm{e}}$-XIV ${ }^{\mathrm{e}}$ siècles, qui se traduit par les très nombreuses constructions de châteaux ${ }^{5}$, a pu favoriser la demande du marché local. Tout comme l'abbaye de Sturzelbronn, Villers-Bettnach ${ }^{6}$ contrôlait un important patrimoine d'étangs, aujourd'hui en grande partie disparu. Ce dernier s'établissait sur le cours amont de la Canner. À l'ouest de la Lorraine, l'abbaye de Saint-Benoît-en-Woëvre ${ }^{7}$ est à l'origine de l'aménagement de la « Voivre ». Ce toponyme désigne une forêt marécageuse occupant la dépression au pied des Côtes de Meuse. L'abbaye, pour se mettre hors d'eau réalise d'importants travaux hydrauliques sur l'Yron et installe une digue de 300 mètres de long et de 2 mètres de hauteur en amont de l'abbaye. Dans ce cadre géographique de la Woëvre l'aménagement d'étangs permet de reconfigurer le réseau hydrographique, de drainer et de bonifier les terres situées en aval.

7 Dans la Woëvre, les aménagements d'étangs correspondent à un phénomène de colonisations agraires encouragées par les comtes de Bar au XIII ${ }^{e}$ siècle. C'est dans ce contexte qu'est réalisé l'étang de Lachaussée mis en eau en 1273 et exploité par les moines de Saint-Benoît. L'étang est lié à la création du village de Francheville-laChaussée. D'autres types d'établissements religieux ont également développé un savoirfaire en matière de pisciculture et d'étangs. Les Templiers puis les Hospitaliers de Gelucourt disposaient en marge du domaine ducal du Lindre de près d'une dizaine d'étangs (Mathis, 2009).

8 La plupart du temps, lorsqu'il n'y a pas de grands établissements religieux pérennes, l'origine des étangs apparait bien plus obscure. L'histoire médiévale des étangs de Lorraine reste à écrire, et tout particulièrement celle des grands étangs mosellans du Lindre, du Stock, de Gondrexange, de Mittersheim. La tradition de leur mise en eau renvoie à de multiples acteurs (évêques de Metz, abbayes bénédictines, barons de Fénétrange, ducs de Lorraine...). Les sources historiques restent vagues, et quand elles existent, ne permettent pas d'évaluer l'état de réalisation de l'étang. En effet, la construction de ces lacs-étangs reste un processus lent, par extension de propriétés et par coalescence de « cornées $»^{8}$, constituant ainsi de grands plans d'eau. Il est probable qu'au 
fur et à mesure les chapelets d'étangs n'aient plus formé qu'un seul étang. Ces réalisations sont conditionnées par la maîtrise technique de grandes digues-barrages qui obstruent le cours d'eau. C'est probablement vers le XIII ${ }^{\mathrm{e}}$ siècle que ce seuil est atteint si on considère la mise en eau de Lachaussée. Toutefois, ce seuil technique est peut-être plus tardif pour le Lindre où la chaussée-barrage est établie directement sur la Seille, et pour l'ancien étang Griffon à Lagarde où la digue obstruait le Sânon. Il faut considérer que la mise en eau des étangs est un lent processus de conquête de l'amont vers l'aval des vallées. Ce phénomène de conquête s'organise au fur et à mesure de la maîtrise des écoulements des ruisseaux et rivières.

\section{B. Un patrimoine hétérogène plus récent}

9 Conjointement à la vocation agro-piscicole des étangs qui constitue en grande partie l'usage de l'eau en "plaine », s'est développée une activité proto-industrielle liée aux activités forestières (flottages, scieries, verreries, papeteries, forges...) dans le massif des Vosges et de la Vôge. Il s'agissait d'assurer une réserve d'eau suffisante pour permettre la continuité de l'activité sylvicole ou industrielle. Ainsi, dans le massif des Vosges du Nord, les flotteurs vont établir des étangs temporaires sur la Sarre, la Zinsel, la Zorn ou la Horn pour assurer sur des cours d'eau aux débits très modestes le départ des trains de flottage du bois (Husson, 1991 ; Kleine, 2003 ; Jéhin, 2005). Le système de flottage s'est établi également en plaine autour des salines du Saulnois, mobilisant les ressources en eau d'étangs afin d'assurer là encore le transport des bûches (Mathis D. et A., 2013).

10 La ressource en eau que représentent les anciens étangs a été nécessaire pour assurer le fonctionnement du réseau de canaux (Canal de la Marne au Rhin, Canal des Houillères), notamment dans les secteurs inter-fluviaux. Ainsi, les étangs de Mittersheim, Gondrexange, ou encore du Stock sont traversés par les canaux. Le niveau d'eau des étangs a été rehaussé afin d'assurer une réserve d'eau destinée à franchir l'interfluve entre les bassins de la Sarre à l'Est et de la Meurthe à l'Ouest.

11 La vocation militaire des étangs est relativement ancienne et n'est pas propre à l'espace lorrain. La première mise en œuvre d'un dispositif est liée au domaine du Lindre et concerne la vallée de la Seille. Pour Vauban, les eaux de l'étang devaient permettre d'inonder la vallée de la Seille, isolant la place forte de Marsal. Ce dispositif devait également servir pour la défense de la place de Metz. Si en 1870, le Grand Étang était en assec cultural, en revanche en août 1914 et durant l'automne 1944, les eaux de l'étang inondèrent la vallée de la Seille. Cette technique des inondations fut également mise en œuvre dans la réalisation de la «Ligne Maginot aquatique». Il s'agit d'un vaste projet d'aménagement et de création d'étangs, réalisé en 1935, dans le secteur fortifié de Forbach (entre Nied et Sarre). Ces masses d'eau étaient destinées, lorsqu'elles étaient vidangées, à «tendre des inondations » devant une progression ennemie. De cette façon, une vaste coupure d'eau pouvait être réalisée et maintenue grâce à un ingénieux système de seuils, de "portes" et de barrages. Ce système fortifié inondable impliquait la réalisation de cinq grands étangs, mais aussi l'utilisation des étangs du Stock et de Mittersheim dont les eaux devaient se déverser par le Canal des Houillères vers la Sarre. La digue de l'étang du Stock a été relevée, portant sa superficie à 750 hectares et entrainant la mise sous l'eau d'une partie du village de Rhodes. Les vallées du Rothenbach et du Schwarzbach dans le Pays de Bitche furent également aménagées en zone fortifiée inondable avec une succession de douze barrages-portes. Le déclassement, puis la 
« démilitarisation » de ces cours d'eau, ne remettent pas en cause la présence des étangs. Ces paysages d'eau hérités sont désormais intégrés à l'identité et à l'habiter des populations, produisant ou enrichissant de nouveaux pays d'étangs tels le Pays de l'Albe et des Lacs (Chiffre et Mathis, 2013).

La mise en eau du lac de Madine en 1965 souligne toujours la dynamique paysagère de production des zones d'étangs dans la Woëvre. Le lac devait servir de réserve d'eau potable pour l'agglomération messine. Il devait également devenir une réserve de chasse et de faune sauvage et pour finir un espace de loisirs au sein du PNRL.

13 Ainsi, si les systèmes agro-piscicoles de plaine ont décliné, les étangs ont trouvé de nouveaux usages et de nouvelles fonctionnalités. L'abandon fonctionnel partiel des systèmes d'étangs durant les $\mathrm{XIX}^{\mathrm{e}}$ et $\mathrm{XX}^{\mathrm{e}}$ siècles a permis l'émergence de nouveaux usages qui ont fini par s'imposer.

\section{Glissement fonctionnel des vieux terroirs d'étangs}

Grande région de pisciculture, la Lorraine n'a pas véritablement de culture ni d'identité d'étangs, c'est en partie l'explication du déclin de la pisciculture des étangs à partir du $\mathrm{XIX}^{\mathrm{e}}$ siècle. En effet, l'exploitation des étangs a longtemps pesé sur des communautés villageoises qui ont cherché, dans la mesure du possible, à en limiter les contraintes (Mathis, 2009). Pourtant, après la Révolution, le déclin de l'activité piscicole n'a pas entrainé systématiquement l'effacement d'étangs. En fait, ils sont restés des éléments du paysage agraire, exploités par une société de grands propriétaires fonciers issus de la bourgeoisie urbaine venus se mettre "au vert», chasser et pêcher. Les projets territoriaux centrés sur les étangs sont nés tardivement et sont souvent encore en voie de consolidation comme l'a souligné D. Mathis avec le Pays des Étangs (2014). Les dynamiques agraires et paysagères qui ont marqué l'après Seconde Guerre mondiale ont souligné la fragilité de l'héritage lentique. Ce dernier devait évoluer dans ses fonctionnalités afin d'entrer dans une phase de préservation des terroirs d'étangs. De multiples acteurs tant institutionnels que privés ont pris en charge cette mutation.

\section{A. L'activité piscicole : entre tradition et redynamisation}

15 La pisciculture lorraine a reposé sur d'imposants domaines d'étangs constitués par le regroupement de multiples étangs autour des grands lacs-étangs de Lorraine (Stock, Gondrexange, Lindre, Mittersheim, Lachaussée...). Une organisation rationnelle des pêches avec des étangs d'alevinage plus modestes, situés à la périphérie du domaine, est destinée à fournir les alevins nécessaires à l'empoissonnement des grands étangs. Jadis, l'exploitation de ces domaines d'étangs mobilisait une main-d'œuvre nombreuse à l'échelle d'importantes seigneuries regroupant plusieurs villages, répartissant les corvées. Ces dernières concernaient l'entretien de la digue et des berges, les activités de pêche, le charroi, l'alevinage... Le matériel de pêche était conservé dans une " tour » qui commandait la chaussée. Le cycle piscicole prévoyait également un assec cultural destiné à lutter contre l'atterrissement et l'eutrophisation. Le domaine du Lindre constitue à ce titre un modèle (Mathis, 2009 ; 2010). Aujourd'hui, la Lorraine avec 1100 tonnes de poissons récoltés est la troisième région française de production de poissons d'étangs. 
Le maintien des activités piscicoles, avec la mise en assec destinée à la pêche automnale, constitue toujours un temps fort dans le cycle des étangs (fig. 2). Les " grandes pêches " de l'étang de Lindre ou de celui de Lachaussée sont les vitrines de cette activité. Le Domaine du Lindre est le premier centre piscicole de France. Il produit près de 80 tonnes de poissons par an. La vidange des étangs et la pêche traditionnelle au filet constituent un temps fort dans le cycle des étangs. Elles témoignent de la résistance fonctionnelle des activités piscicoles. Toutefois, il est nécessaire d'y associer des activités complémentaires. Ainsi la «Grande Pêche » du Lindre draine environ 13000 visiteurs en deux jours. Le Conseil Général de la Moselle, propriétaire du site, en fait un grand événement pour le village de Lindre-Basse (228 habitants en 2012). La vente directe de poissons au centre de pisciculture du Domaine, le spectacle de l'assec et de la pêche au filet présentent une activité « authentique » et justifient aux yeux du public l'acquisition et les mesures de protection du milieu stagnustre. À ces journées de pêche est associée une politique de valorisation des terroirs, notamment autour du « Manger mosellan » et en liaison avec les anciens terroirs viticoles délimités par « l'AOC Moselle » (Mathis, Chiffre, 2015).

Figure 2. Le Domaine du Lindre (Saulnois) principal pôle piscicole de France.
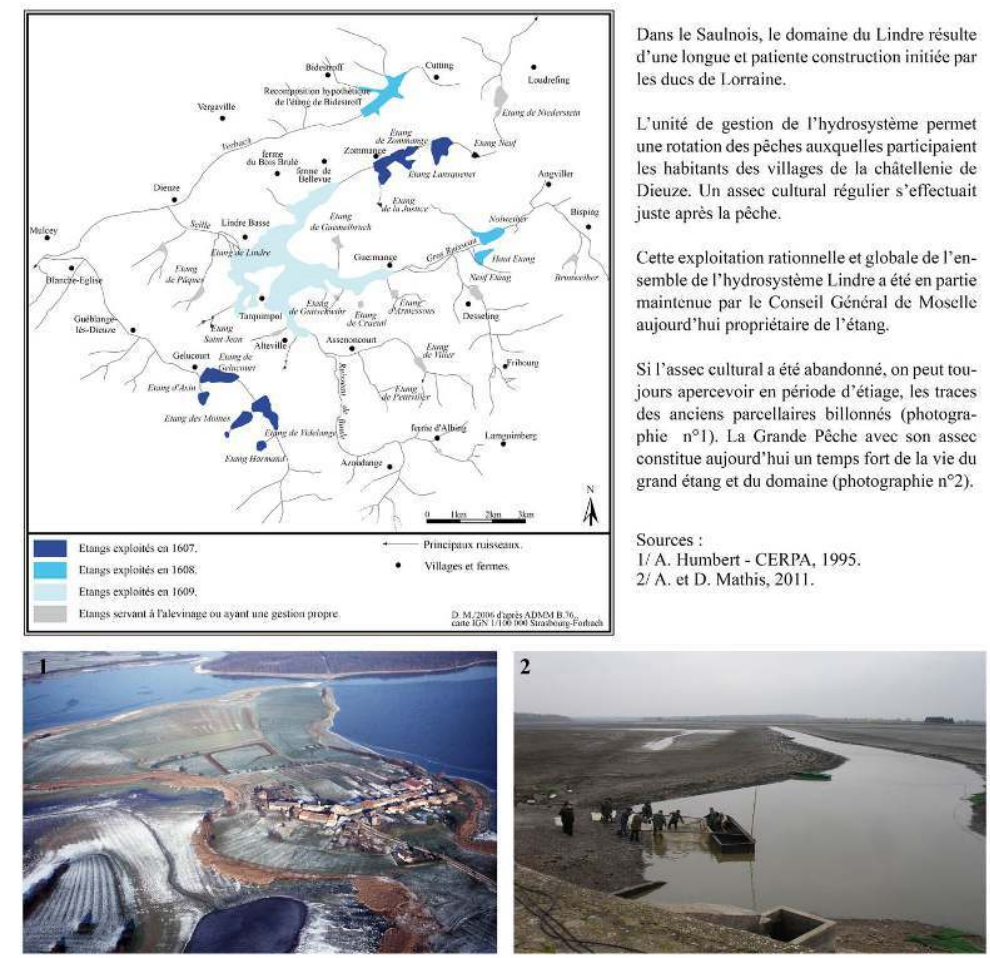

À partir du savoir-faire acquis dans la gestion du Domaine du Lindre, le département de la Moselle s'est lancé avec de nombreux partenaires (Conservatoire d'Espaces Naturels de Lorraine (CEN), PNRL, Établissement Public de Coopération Intercommunale (EPCI)) dans la définition d'une pisciculture extensive visant à assurer une gestion rationnelle des étangs entre activités piscicoles, loisir-détente et conservation-protection. Cette politique est reprise lors de l'acquisition en 2011 par la Communauté de Communes du Centre Mosellan (CCCM) de l'étang du Bischwald. Elle permet de maintenir l'activité de pêche. Certes le site doit être aménagé pour accueillir des visiteurs et des travaux sont envisagés afin de limiter l'impact de la vidange en aval, avec notamment les risques de conflits 
d'intérêts. Ils concernent le ruisseau du Bischwald dans sa section traversant la base aérienne de Bistroff-Grostenquin (concertation fin 2012 entre l'Armée, la Communauté de Communes du Centre Mosellan (CCCM), les exploitants agricoles riverains, le pisciculteur et le CEN Lorraine). La mise en œuvre d'une véritable filière de transformation reste à l'étude. En Meuse, le site de Lachaussée pratique depuis longtemps ce cycle d'activité. Ce modèle, inscrit dans la Petite Woëvre, constitue un site d'appel pour la pisciculture meusienne. Les problèmes d'organisation sont les mêmes que dans l'est mosellan, à savoir trouver un équilibre entre exploitation des étangs et préservation de l'environnement stagnustre. Les réflexions actuellement menées par la Région Lorraine soulignent l'importance de la question piscicole et la redéfinition fonctionnelle du patrimoine lentique lorrain.

\section{B. Le difficile équilibre entre constructions touristiques et protection}

La mise en œuvre de la politique des Parcs Naturels Régionaux a induit une «distribution spatiale des loisirs » au sein de l'espace rural. Ainsi comme l'a souligné Y. Le Caro (2012), il s'agit d'un "assolement récréatif ». En effet, comme le fait remarquer D. Mathis (2014) à propos des lacs-étangs du Stock, de Mittersheim ou de Gondrexange, «les grands étangs constituent une ressource paysagère à préserver tout en étant l'élément attractif créant également un espace convoité. ». Espace naturel convoité, les rives des étangs attirent de manière plus ou moins spontanée des reconversions touristiques et de loisirs. Elles sont souvent nées de ce double déclin des systèmes piscicoles et agricoles.

En effet, le faible coût du foncier agraire situé à la périphérie de ces masses d'eau a permis l'essor de structures multiformes. Ainsi, les berges ont été colonisées par des campings résidentiels, des lotissements de résidences touristiques, des cabanons de pêches... Ces constructions ont été parfois spontanées et non permanentes. Ainsi sur des petits lots relevant d'une copropriété, les résidents posent et installent une caravane ou un bungalow. Cet habitat «mobile » est consolidé par l'ajout de terrasses, de haut-vents, de murets, puis il connaît une intégration progressive dans de nouvelles constructions qui font disparaitre l'habitat originel désormais induré. Ce processus de consolidation et de sédentarisation de l'habitat mobile de vacances est une forme de construction qui s'effectue hors de toutes règles d'urbanisme. Outre le problème de ce déficit d'urbanisme, le processus pose la question de l'absence de réseau d'assainissement et de rejets des eaux usées dans les étangs. Aujourd'hui, la normalisation de ces espaces est devenue une priorité pour les communes ou pour les communautés de communes qui ont hérité de cette compétence. À ces formes spontanées et désormais consolidées se sont agglomérés des quartiers résidentiels plus élaborés, associés à des bases de loisirs avec plages, pontons nautiques ou de pêches. Ils s'accompagnent d'une mise en valeur de la berge (fleurissement, mobilier urbain, allée piétonne...). Les berges des étangs, ainsi aménagées, copient finalement les fronts d'eau des littoraux touristiques (fig. 3). 
Figure 3. L'urbanisation des rives de l'étang du Stock (Pays des Étangs).

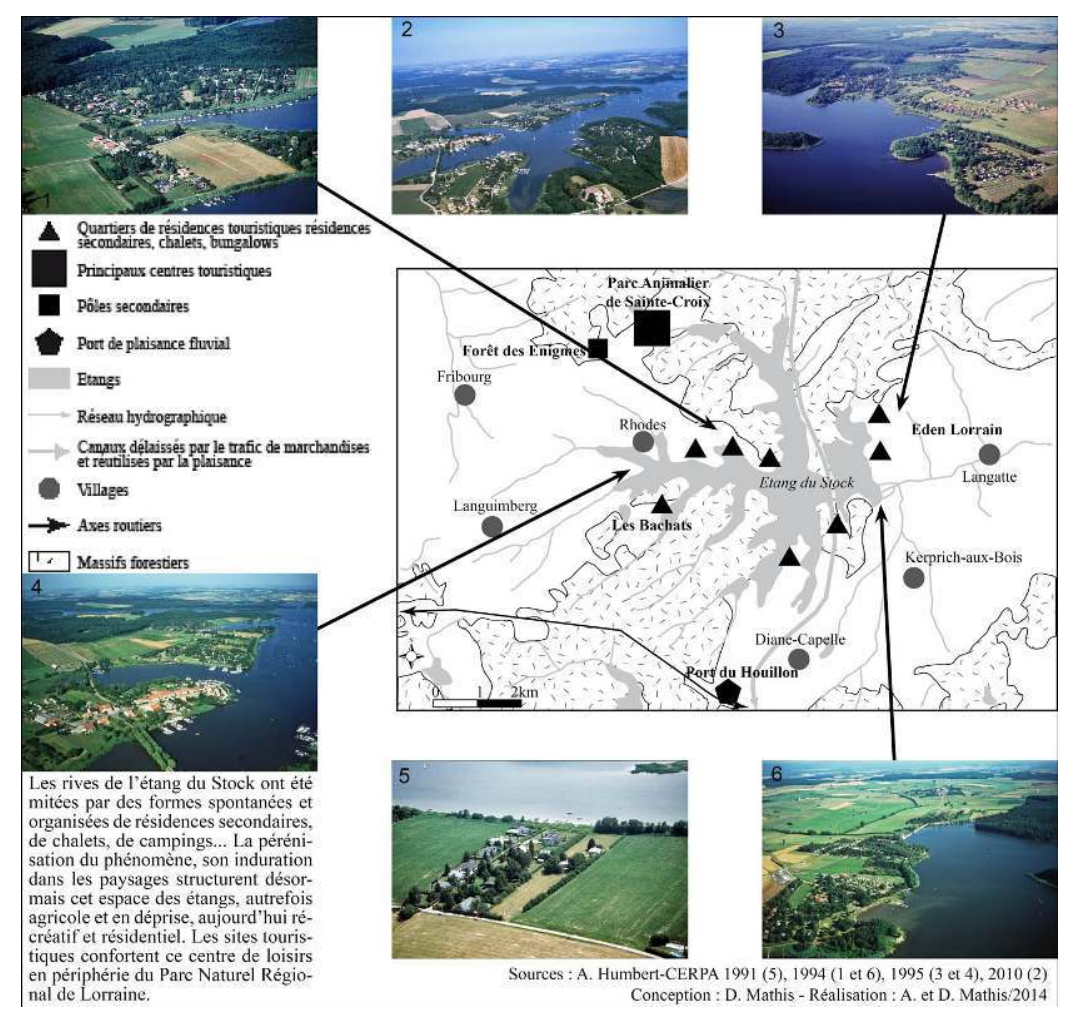

Par toutes ces formes, l'urbanisation des rives des étangs donne naissance à des fronts d'eau résidentiels et touristiques tournés vers l'étang. Le paysage d'eau est devenu un facteur majeur d'aménagement de l'espace rivulaire. S'exprime finalement le «désir de rivages " rapporté aux espaces stagnustres et lacustres. On peut toutefois distinguer deux types de paysages rivulaires. D'une part, l'urbanisation résidentielle avec front d'eau, responsable de l'aménagement urbain des berges, et d'autre part le paysage rivulaire aménagé par les pontons avec cabanons de pêche. Ces derniers mordent sur l'étang, enjambent la zone bordière de la roselière. De cette façon, cet espace d'intérêt écopaysager peut être conservé. Ces aires de naturalité, que représentent les étangs, ont été rapidement convoitées par une population urbaine issue de la Grande Région (SarLor-Lux). Afin de préserver les étangs d'une urbanisation touristique, les différents acteurs du développement local, tels que les parcs naturels régionaux, les communautés de communes, les départements et la région Lorraine ont d'abord mis en œuvre une politique de préservation des zones humides et des étangs. Elle s'est notamment manifestée par l'achat de domaines stagnustres permettant leur conservation'. Ainsi, dans le cadre de la définition de projets territoriaux, ces acteurs ont, dans un premier temps, insisté sur la préservation qui s'est ultérieurement prolongée par une démarche de valorisation. Cette dernière cherche à intégrer les populations locales aux touristes et aux visiteurs.

21 La protection et la mise en réserve des zones humides expliquent les projets du lac de Madine ou l'achat par le Conseil Général de la Moselle du Domaine du Lindre. La logique est avant tout celle de la protection des espaces stagnustres. L'urbanisation de l'étang du Stock et le projet touristique qui en résultent sont alors montrés comme l'antithèse de l'aménagement territorial. Au fur et à mesure que la législation a été établie, les aires de protections se sont surimposées aux étangs, notamment pour les étangs d'Amel, le Lindre 
ou Lachaussée. Il s'agit alors de construire des îlots protégés au sein même du PNRL mais aussi au sein du Parc Naturel Régional des Vosges du Nord puis, par extension, autour de nouveaux lieux, tel le site Natura 2000 «Plaine et Étang du Bischwald ». Ce dernier couvre 2481 ha, avec en son cœur le site protégé «Étang et prairies du Bischwald» (258 ha). Cette politique correspond à la logique environnementale de l'îlot. Finalement les étangs, avec leur forte image de naturalité, sont aujourd'hui l'épicentre de cette politique. Toutefois, cet empilement de statut de protections porte en lui le risque d'étouffer toute mise en valeur touristique, même lorsque cette dernière est associée au projet territorial raisonné et soucieux de ménager l'environnement. Ainsi, pour Madine, protégé en plus par la « loi littoral », la base touristique avait du mal à renouveler son offre. En 2013-2014, le Conseil Régional de Lorraine a entamé une première tranche de travaux investissant 14,5 millions d'euros pour réaménager les berges et la plage de Nonsard. Les possibilités du port de plaisance sont portées à 380 bateaux. Un « front d'eau » de promenade de 800 mètres de long borde le lac, avec en champ panoramique la ligne des côtes de Meuse et le site de la Butte du Montsec. De nouvelles cellules commerciales, un restaurant, des bars et un office de tourisme accompagnent cette modernisation. L'objectif est d'attirer 350000 visiteurs, contre 150000 actuellement. De multiples activités de plein air (équitation, accrobranche, sentiers de découvertes, observatoires...) doivent compléter l'aménagement. Une seconde tranche de travaux d'un investissement de 15,5 millions d'euros, prévoit de reconfigurer l'ensemble de l'offre d'hébergement. En fait, le projet Madine s'inspire de la réussite touristique de l'Étang du Stock et du Parc animalier de Sainte-Croix, tout en conservant le volet environnemental mis en place. Madine souligne le glissement d'un développement durable des sites d'étangs autour d'un projet exploitant la valeur des paysages d'étangs.

\section{La valeur des paysages d'étangs}

Bien que secondaire dans l'organisation des paysages agraires, l'étang est aujourd'hui une des traces les plus marquantes de ces anciens paysages. Vestige déclassé des anciens stagno-systèmes, il a perdu partiellement ou totalement sa fonction productive, supplantée désormais par leur valeur naturelle, paysagère et « sitologique ». Cette valeur sensible du paysage est liée à une triple symbolique :

- celle de l'étang, masse d'eau stagnante, calme, immuable et porteuse de mélancolie,

24 - celle de la nature préservée et protégée,

25 - celle d'un espace archaïque intemporel, authentique.

26 L'objet étang illustre le glissement sémantique du paysage agraire au paysage naturel. Considéré pour sa valeur environnementale et écologique, il a perdu sa valeur fonctionnelle. Le milieu stagnustre offre une image authentique et symbolique de calme et d'intemporel. La démarche entreprise par le PNRL a été d'assurer la préservation de ces espaces, mais aussi la valorisation en lien avec une patrimonialisation « sitologique ». La sensibilisation des populations à la valeur paysagère, patrimoniale et environnementale des étangs a permis d'assurer leur pérennité mais aussi de redonner à ces espaces un rôle économique tourné vers un usage raisonné de ces masses d'eau pour les multiples acteurs investis. Appuyé sur la valorisation des activités touristiques et récréatives, l'essor qui vient d'être expliqué a donné naissance à une activité encore souvent artisanale et spontanée. La concrétisation récente du projet de Center Parcs (Groupe Pierre et Vacances) souligne l'évolution actuelle d'une activité touristique artisanale vers une 
activité structurée. La reconfiguration du site touristique de Madine souligne également ce phénomène. L'aspect patrimonial de l'étang est désormais au cœur des démarches territoriales. Ainsi comme le soulignent M. Périgord et G. Donadieu, « il s'agit donc pour les acteurs d'un territoire de mettre en œuvre un processus, déclenché par une commande publique, qui vise à maintenir les continuités spatiales et temporelles des territoires vus et vécus. Sa finalité est autant esthétique (le plaisir contemplatif) que fonctionnelle (créer ou restaurer des utilités) » (2012, p. 93).

\section{Anciens et nouveaux étangs au cœur des démarches territoriales et environnementales}

Désormais, les enjeux de la préservation de ces anciens terroirs constituent une question d'avenir pour les acteurs locaux, en particulier les collectivités territoriales qui ont privilégié une utilisation rationnelle des zones humides. Cette dernière associe à la préservation écologique de ces terroirs, des formes de pisciculture extensive. Cette politique est promue par les départements de la Moselle ou de la Meuse. Toutefois, la démarche de préservation/valorisation de ces anciens terroirs d'étangs se conjugue aujourd'hui avec l'essor de nouveaux territoires d'étangs en situation périurbaine ou urbaine et nés de la réhabilitation des gravières et des sablières dans les grandes vallées alluviales. Si les formes d'utilisation restent comparables, elles aboutissent à l'essor de nouvelles dynamiques des terroirs d'étangs.

\section{A. Les nouveaux terroirs d'étangs par la valorisation des carrières des granulats alluvionnaires}

Depuis la seconde moitié du $\mathrm{XX}^{\mathrm{e}}$ siècle, les paysages des grandes vallées lorraines (Meurthe, Moselle, Meuse ou Sarre) ont été marqués par l'extraction des granulats et la reconversion spontanée ou volontaire de ces carrières ennoyées en plans d'eau. À l'origine, ces sites n'ont pas fait l'objet d'une reconversion particulière, la friche industrielle "cicatrisant » lentement et évoluant vers une renaturation «sauvage». Il s'agit alors « d'un non-lieu » dont la qualité est jugée faible, d'où parfois le comblement de ces carrières et l'absence de souci de valorisation. L'évolution des contraintes légales de remise en état du site, rappelée par S. Edelblutte (2000, p. 301) entraîne un retraitement de plus en plus complexe et un réemploi fonctionnel immédiat. La concentration des entreprises de ce secteur industriel a uniformisé la gestion et les techniques de renaturation. Le retraitement des zones d'extraction entre dans un processus accéléré, le "génie végétal " permettant de reconstruire un environnement écologique. Ainsi, comme C. Carré et M. Charrier (2002)l'ont évoqué dans leur étude de cas pour l'île-de-France, le retraitement reste un « aménagement des nuisances ».

Dans l'espace lorrain, l'Agence de l'eau et/ou le CEN Lorraine tentent de préserver les fonds de vallées de la "prédation » des carristes. Ainsi, pour la vallée de la Moselle, ces exploitations "engendrent une dégradation du fuseau de mobilité en créant des points "durs" incompatibles avec la mobilité du cours d'eau. C'est toute la dynamique fluviale qui est alors modifiée $»^{10}$. D'autre part, les carrières ennoyées prennent la place des prairies humides qui bordent la rivière. 
30 Le réaménagement des anciens sites d'extraction «construit » des plans d'eau dont la gestion des contraintes d'écoulement implique un fonctionnement en chapelet proche des systèmes d'étangs établis sur les cours d'eau. Sur de nombreux secteurs de la Moselle ou de la Meurthe, le lit mineur se trouve bordé et accompagné par un couloir « d'étangs ». À ce titre, il s'agit d'un nouveau faciès et d'un nouveau terroir. Le retraitement paysager produit des endiguements géométriques, ourlés de plantations ou réoccupés par une végétation colonisatrice. Son impact est finalement assez identique à celui des étangs plus anciens. Toutefois, selon que le regard porté sur ces exploitations se focalise sur la morphologie fluviale, les paysages produits par la reconversion paysagère et le «génie végétal » ou le recyclage fonctionnel, la valeur de ces anciennes friches industrielles est différente (fig. 4).

Figure 4. Les paysages d'étangs hérités de l'exploitation des anciennes gravières.
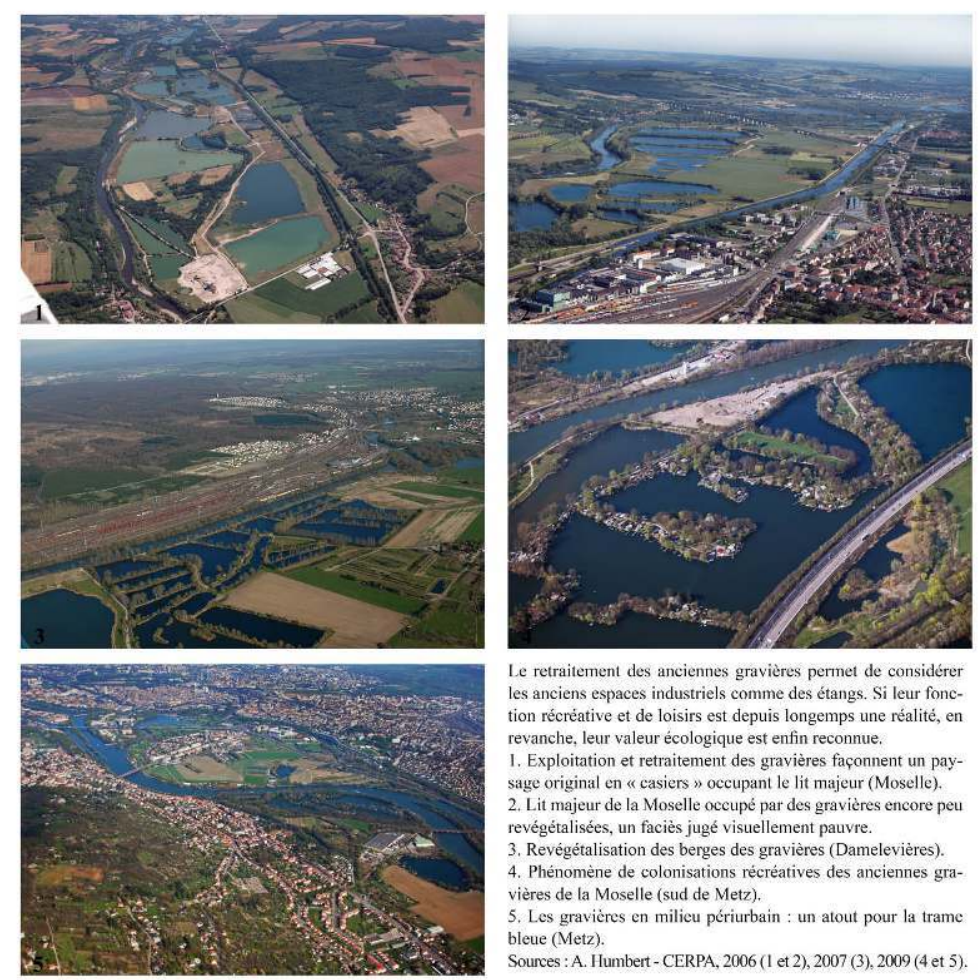

La fonctionnalité de ces étangs a très vite été trouvée. Espaces de pêche, de loisirs et de détente, ils s'inscrivent à ce titre dans le même processus de transformation des vieux étangs. Très tôt donc un «nouvel assolement récréatif » réoccupe les anciennes carrières, le plus souvent de façon plus ou moins spontanée. Ces étangs s'inscrivent dans les espaces urbains et périurbains. Le « désir de rivage » se retrouve dans les aménagements de ces plans d'eau qui intègrent également la construction des «fronts d'eau ». L'aménagement de ces espaces d'eau semble être préféré à l'aménagement des bords de rivières : sentiers pédestres destinés à la promenade, aires de jeu, de pique-niques, plages et zones de baignade, jeux nautiques (Mathis, Chiffre, Harmand, 2015). En effet, l'eau des étangs paraît calme, offre une sérénité et surtout ne connaît pas, comme les grandes rivières lorraines, de crues régulières. Le schéma d'appropriation de ces étangs-gravières par les populations urbaines et périurbaines relève du même processus que celui qui s'est opéré durant les années 1950-1970 pour les vieux étangs. En effet, il s'agit d'une démarche de 
recherche de naturalité, non plus uniquement de fin de semaine ou de villégiature, mais désormais quotidienne. Ce besoin de "nature » évolue également et peut être qualifié de «droit à la nature ». C'est ce qui tend à faciliter l'effacement de la frontière entre ville et campagne. La nature retrouve, au sein des espaces urbains et périurbains, une place nouvelle et les étangs-gravières se transforment de «non-lieu » en "lieu de la ville ». Ainsi, autour de Metz, les anciennes gravières ont été appropriées en lieux de détentes avec lotissements de ponton de pêche ou de cabanons parfois très hétéroclites. Ces constructions, comme dans l'étude de cas des "vieux pays d'étangs", s'effectuent de manière spontanée et parfois en dehors de toutes règles d'urbanisme. Toutefois, la contrainte de retraitement de ces friches industrielles par les carriers oblige ceux-ci ainsi que les communes à construire un projet concerté. Il s'agit d'une seconde phase plus institutionnelle qui est mise en œuvre avec la réalisation de projets plus aboutis. Espaces de détentes, bases de loisirs et de tourisme se développent avec plages, lieux de baignades, terrains de jeux, aires de pique-nique, campings et villages de gîtes (Lac de Saulxures-sur-Moselotte, bases de loisirs de Damelevières, de Favières, Lac de Contrexéville...).

32 À Sarrebourg, "l'étang Lévêque » a été intégré au complexe sportif de la ville (piscine, stade) et offre un potentiel pour les jeux d'eau (kayak, plages, baignades...). Ce lieu de loisirs et de détente créé autour de cette ancienne sablière reconvertie en étang constitue un espace public important. C'est un lieu de vie où les habitants de la ville, mais également des ceintures périurbaines, viennent pratiquer une activité sportive ou simplement se détendre. La taille modeste des gravières, les berges plates, les réaménagements paysagers (arbres ornementaux, peupleraies, haies...) sont autant de facteurs favorisant la construction d'un espace public facilement " habitable » par des populations urbaines (ou périurbaines) en recherche d'un lieu de la ville disposant d'une valeur environnementale. Toutefois, ce paysage stagnustre "urbanisé » constitue une pâle copie du paysage des étangs ruraux. Lieu de la ville, ces étangs constituent une transition entre l'espace urbain et la campagne, et sont de plus en plus perçus comme élément naturel. Ainsi à Sarrebourg, l'étang s'insère dans un parcours de découverte des zones humides associé aux rives de la Sarre. C'est dans ce cadre qu'il faut réévaluer la valeur écologique des étangs-gravières. Cette dernière, après avoir été longtemps minorée, est aujourd'hui revalorisée. Ainsi comme l'a souligné A. Guérin-Chapel (2011) pour la Bretagne, «les sablières peuvent être considérées comme des milieux «vierges" où peut se développer un panel faunistique et floristique. La biodiversité qui se développe sur les sites peut être appelée "pionnière». Les caractéristiques morphologiques, climatiques et géologiques falaises minérales, petits plans d'eau, bassins de décantation, surfaces minérales s'échauffant rapidement - sont propices à l'installation de cette biodiversité ». Le retraitement des anciennes gravières en étangs a été souvent purement fonctionnel et destiné à assurer la fixation des berges. Les plantations de peupleraies semblaient de peu de valeur écologique, longtemps considéré comme des milieux médiocres, voire pauvres en termes de biodiversité en raison d'un retraitement banal et presque immuable. Son impact était jugé négatif sur les milieux humides notamment par la désoxygénation due à la libération de poly-phénols lors de la décomposition de la litière. La fonction de cette populiculture est aujourd'hui à nuancer. Comme l'ont souligné M. Zapater et al., (2010), le peuplier impacte moins négativement que les autres espèces rivulaires la qualité de l'eau et notamment en terme de désoxygénation. D'autre part, bien qu'ayant une capacité moins importante "qu'une ripisylve de saule, la peupleraie a un pouvoir dénitrifiant 20 fois supérieur à la prairie» (2010, p. 161).Ce nouveau terroir permet de lutter contre l'eutrophisation et 
son impact sur les cours d'eau est finalement bien moindre que lorsque les étangs sont construits au fil de l'eau. Ce faciès permet également de lutter contre l'échauffement, la régulation/dérégulation des cours d'eau, l'eutrophisation, les interactions concernant la charge alluvionnaire du cours d'eau. La perception de la valeur écologique des étangsgravières évolue donc également dans le cadre de l'aménagement des trames vertes et bleues et des continuités écologiques. L'exploitation des granulats des fonds de vallée a donné naissance à de nouveaux plans d'eau dont le retraitement paysager sécrète de nouveaux terroirs d'étangs prometteurs.

\section{B. Îlots et corridors : quelle logique environnementale pour les anciens et les nouveaux terroirs d'étangs?}

Les étangs lorrains, et plus particulièrement les grands étangs, ont été au cœur d'une démarche de protection et de préservation des milieux stagnustres avec comme priorité la protection de la biodiversité. En quelque sorte, les étangs ont été à la base de la construction des logiques environnementales. À partir de ces îlots, tels que le Lindre ou l'étang de Lachaussée, s'est mise en place une démarche originale qui vise à une " utilisation raisonnable et raisonnée de la zone humide ». Ce souhait a animé la mise en place du territoire des PNRL. Afin de garantir ces « îlots », un empilement de périmètres d'inventaires ou de préservation (ZNIEFF, Ramsar, ZICO...) s'est mis en place, constituant une ceinture de protection. Autour d'une démarche parfois hésitante, les multiples acteurs ont essayé de trouver un équilibre, notamment concernant la pratique de l'assec et les enjeux des vidanges nécessaires, de l'entretien des berges (Chiffre, Mathis, 2014). Il s'agit clairement de trouver par la gestion de la zone humide un équilibre pour un milieu perçu comme naturel, mais qui n'est finalement qu'un «jardin d'eau ». Les gestions des roselières, des berges, des mares, de l'assec, des sédiments, de l'eutrophisation, de la qualité de l'eau sont autant d'apprentissages dans le cadre du maintien fonctionnel de l'étang sans véritable fondement économique. Désormais, c'est bien la demande sociale et culturelle qui justifie l'entretien de ce milieu dont l'équilibre n'est dû qu'à l'intervention humaine. En l'absence d'entretien, à plus ou moins long terme, l'étang évolue «naturellement» vers un comblement et une disparition qui modifie l'offre de biodiversité.

Toutefois, dans la logique actuelle de restaurer des corridors entre les îlots, il est nécessaire de rétablir les continuités écologiques, c'est-à-dire d'entrer dans une démarche de renaturation des cours d'eau. Dès lors, si dans ce contexte le CEN Lorraine a participé à la restauration des milieux stagnustres pour les étangs d'Ommeray et de Bru (Donnelay), en revanche, l'ONEMA a pris en charge le rétablissement des continuités écologiques des ruisseaux transformés par la mise en place de la Ligne Maginot Aquatique (Chiffre, Mathis, 2013). La question des étangs n'a pas été tranchée alors qu'il s'agit du principal obstacle à la biodiversité en raison de leurs rôles dans la politique de développement économique et territorial du Pays de l'Albe et des Lacs (fig. 5). En effet, si la logique d'effacement des étangs ${ }^{11}$ tend aujourd'hui à percer dans la pensée environnementale, elle s'oppose à la demande sociale et culturelle développée depuis les années 1960. Si dans le cas des étangs de la ligne Maginot Aquatique cette démarche n'a pas été proposée, en revanche elle a été mise en application dans le Pays de Bitche sur la Bildmuehle avec la disparition de 6 étangs de 2008 à 2012; la disparition des milieux stagnustres étant compensée par le rétablissement des continuités écologiques sur le 
linéaire de la Bidlmuehle. Cette logique d'effacement, inscrite dans le code de l'Environnement, est aujourd'hui mise en œuvre à titre expérimental et entre en contradiction avec la logique ancienne de protection et de préservation des étangs et également avec le rôle des étangs dans une gestion raisonnée de la ressource en eau.

Figure 5. Les étangs du Pays de l'Albe et des Lacs : la question de la renaturation de la Ligne Maginot Aquatique.
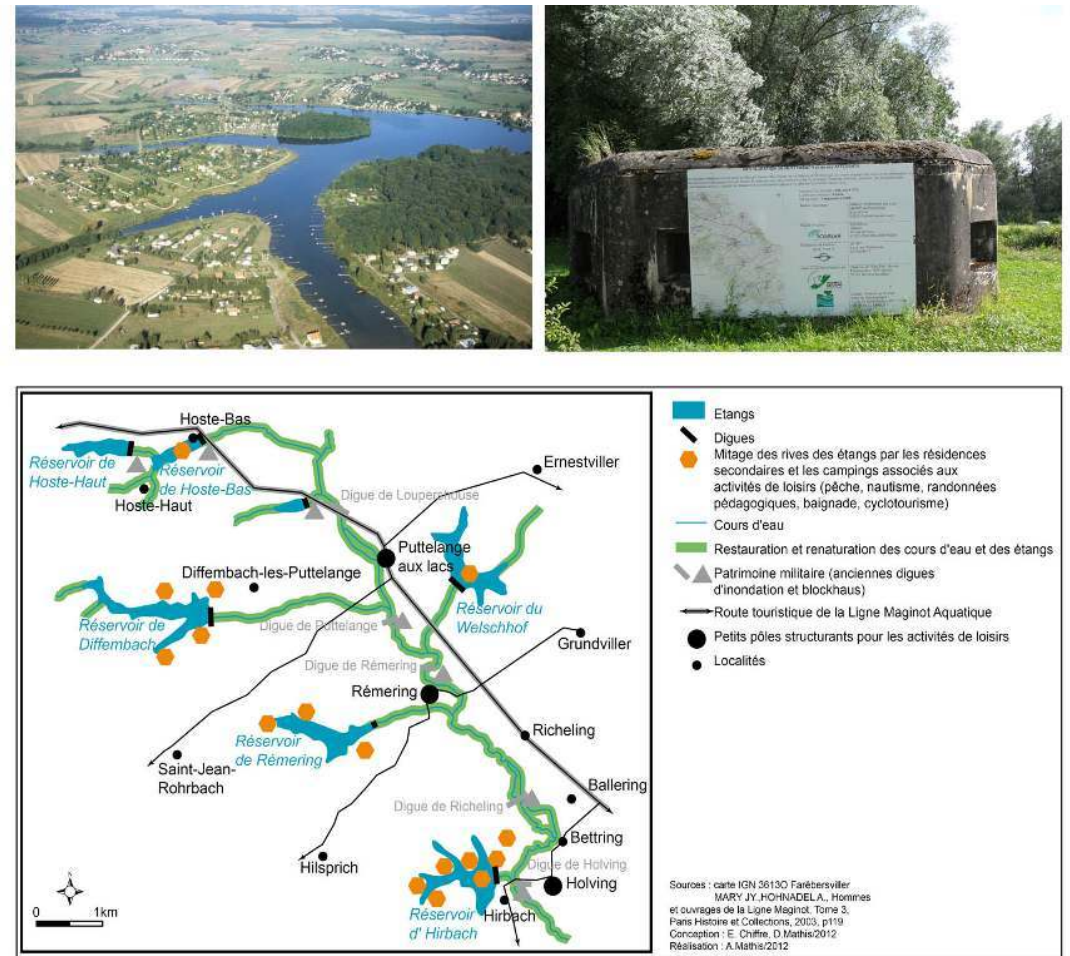

Sources : A. Humbert - CERPA, 1991 et A. et D. Mathis, 2012.

Dans ce contexte, les « étangs gravières » constituent une opportunité pour la mise en place de « corridors écologiques ». Ce paramètre est bien compris et bien inséré dans les espaces urbains des métropoles lorraines. Ces étangs sont plus stables dans leur structure que les étangs traditionnels. Les problématiques de la vidange, de la charge alluviale ou encore de continuité ne se posent pas. La biodiversité qu'ils abritent est aujourd'hui réévaluée, notamment avec les progrès de la restauration «naturelle» ou anthropique mise en œuvre sur les sites des anciennes carrières. Ces étangs facilitent également la gestion écologique des cours d'eau. Aussi dans une logique de corridors écologiques, les étangs gravières lorrains peuvent être un atout dans la définition des trames bleues.

\section{Conclusion}

Le patrimoine lentique lorrain, riche, diversifié, multiforme est confronté aux questionnements actuels du développement durable, de la biodiversité, de la gestion raisonnée des zones humides et de la ressource en eau. Ces étangs dont la valeur économique première est aujourd'hui bien modeste, ont fait l'objet d'un recyclage fonctionnel, opéré suivant une double logique : un assolement touristique et de loisirs et un assolement environnemental et pédagogique. La fonction productive est passée en second plan, même s'il existe toujours une activité piscicole, mais cette dernière ne 
parvient pas réellement à s'organiser en filière et reste à un niveau modeste malgré le soutien actif des conseils départementaux qui sont les principaux acteurs d'une politique volontariste. Cette politique cherche à favoriser la construction d'un système productif piscicole extensif. Ce dernier offre un équilibre entre un assolement piscicole et un assolement récréatif et touristique en tenant compte de la question environnementale. Présenté comme durable, ce système est destiné à assurer la permanence des terroirs d'étangs dans un contexte de disparition de ces derniers ou encore de surexploitation de l'espace stagnustre dans le cadre de projets touristiques et de loisirs. Trouver un équilibre au sein des anciens terroirs et pays d'étangs est une condition vitale pour assurer la pérennité de ces paysages originaux.

Aujourd'hui, la requalification des espaces de gravières et l'évolution de la perception de ce nouveau patrimoine lentique modifient la trajectoire paysagère et fonctionnelle de ces étangs. Si à l'origine l'absence de projets de reconversion a permis la colonisation de ces berges par les activités de loisirs et de détente, désormais la prise en compte de ces espaces par les communes a structuré cet assolement. Aujourd'hui, les progrès des requalifications paysagère et environnementale ont transformé cet héritage peu valorisé en un lieu dont la valeur est de plus en plus reconnue, particulièrement dans le cadre périurbain et urbain. Les anciennes gravières, retraitées en étangs sont considérées comme un atout dans la mise en œuvre de la trame verte et bleue, faisant suite à la mise en place de "fronts d'eau» de loisirs au sein des grandes agglomérations. Ainsi, les étangs-gravières retrouvent une place au sein du recyclage d'un « espace nature » ayant un fort potentiel.

Toutefois, les enjeux de continuité écologique, notamment les réflexions qui concernent l'effacement des étangs, annoncent et soulignent aujourd'hui les difficultés pour assurer la permanence d'une politique de gestion durable autour des étangs lorrains. Cette dernière a été fondée sur les géosymboles des grands étangs, vitrines d'une politique de protections et de valorisation au sein de projets de paysages, territoriaux, systémiques et aux fonctionnalités complémentaires.

10. Agence de l'Eau Loire- Bretagne, Etudes sur l'eau, Guide technique inter-agences des zones humides et de la ressource en eau, fiches de cas, consulté le 05 décembre 2015, http://www.eau-loire-bretagne.fr/espace_documentaire/documents_en_ligne/ guides_zones_humides/fiches\%20K.pdf

40 11. Établissement Public du Bassin de la Vienne, « Gestion des étangs : l'effacement, une solution à envisager », consulté le 05 décembre 2015, http://www.eau-loire-bretagne.fr/ les_rendez-vous_de_leau/les_rencontres/rencontres_2010/6-Effacement_etang.pdf, ONEMA, «La suppression ou la dérivation d'étangs sur cours d'eau», consulté le 05 décembre 2015, http://www.onema.fr/IMG/Hydromorphologie/22_0_intro_r2_vbat.pdf, ONEMA, "Effacement d'un chapelet de huit étangs sur la Bildmuehle», consulté le 05 décembre 2015, http://www.documentation.eaufrance.fr/entrepotsOAI/AERMC/ R111/47.pdf 


\section{BIBLIOGRAPHY}

Benarrous R., Marinval M-C., 2006, « La Carpe (Cyprinus carpio), cette orientale qui séduit l'Occident au Moyen Age », Derex J.-M., (textes réunis par), La production des étangs du Moyen Age à l'époque contemporaine, Actes de la journée d'étude 2005 du Groupe d'Histoire des Zones Humides, 120 p., p.9-22.

Bédoucha G., 2011, Les liens de l'eau, En Brenne, une société autour de ses étangs, Paris, co-éditions QUÆ Éditions et Éditions de la Maison des Sciences de l'Homme, collection Natures sociales, 688 p.

Carré C. et Charrier M., 2002, «La gestion d'une ressource non renouvelable, entre gestion durable et aménagement des nuisances, le cas des granulats alluvionnaires en Île-de-France ", Annales de géographie, $111^{\text {ème }}$ année, $n^{\circ} 626$, p.406-418.

Chiffre E. et Mathis D., 2013, « Démilitarisation des cours d'eau de la ligne Maginot aquatique », Revue du Nord, Hors-série archéologie, $13^{\mathrm{e}}$ Rencontres internationales de Liessies, 174 p., p.155-164.

Chiffre E. et Mathis D., 2015, « Prise en compte des hydrosystèmes hérités par les parcs naturels régionaux de Lorraine et du Morvan ",Territoire en mouvement, Revue de géographie et aménagement [En ligne], 25-26 | 2015, mis en ligne le 31 mars 2015. URL : http://tem.revues.org/2764

Edelblutte S., 2000, « "Logique d'exploitation" ou "logique de protection" dans la vallée de la moyenne Moselle. Le paysage révélateur des conflits spatiaux liés à l'extraction des alluvions ", G éocarrefour. Vol. $75 \mathrm{n}^{\circ} 4,2000$. L'interface nature-sociétés dans les hydrosystèmes fluviaux. p.293-304, URL : /web/revues/home/prescript/article/geoca_1627-4873_2000_num_75_4_2481

Guérin-Chapel A., «La biodiversité dans les carrières, une réalité ? Avis des associations naturalistes ",Vertigo - la revue électronique en sciences de l'environnement[En ligne], Regards / Terrain, mis en ligne le 31 mars 2011, consulté le 19 janvier 2014. URL : http:// vertigo.revues.org/10702

Husson J.-P., 1991, Les hommes et la forêt en Lorraine, Paris, Bonneton, 318 p.

Jéhin Ph., 2005, Les forêts des Vosges du Nord du Moyen Âge à la Révolution. Milieux, usages, exploitations, Presses universitaires de Strasbourg, 398 p.

Kleine R., 2003, « Le flottage du bois sur la Sarre aux XVIII ${ }^{\mathrm{e}}$ et XIX ${ }^{\mathrm{e}}$ siècles », SHAL - section de Sarrebourg, $84 \mathrm{p}$.

Le Caro Y., 2012, « Les agriculteurs et le partage de l'espace agricole pour des usages récréatifs », in Papy F., Mathieu N., \& Férault Ch. (dir.), Nouveaux rapports à la nature dans les campagnes, Editions QUÆ, 191 p., p.101-117.

Manceron V., 2004, « La mise en valeur agro-piscicole de l'étang en Dombes. Hiérarchie, complémentarité et dépendance entre gens de l'épi et gens de la carpe ", in Les étangs : Espace de production hier et aujourd'hui, Paris, Groupe d'Histoire des Zones humides, Collection Journée d'étude, 88 p., p.9-15.

Manceron V., 2006a, « Le pays de Dombes et ses mises en image : jeu sur les frontières et quête de reconnaissance », Ruralia, n 18-19, p. 211-231. 
Manceron V., 2006b, « Discordes territoriales : les logiques rivales de la gestion de l'eau dans une région d'étangs française », Géographie, Economie, Société, vol 8 (3), juillet-sept, p.319-327.

Mathis D., 2009, « Géohistoire agraire d'un pays lorrain :le Saulnois », Thèse, Nancy-Université, 966 p. http://docnum.univ-lorraine.fr/public/NANCY2/doc460/2009NAN21021.pdf

Mathis D., 2010, « Le système agricole des étangs : l'exemple du Lindre à l'époque moderne ", Villages Lorrains, $\mathrm{n}^{\circ} 132$, automne 2010, p.24-28.

Mathis D., 2014, «Recomposition territoriale d'un espace rural flou : l'exemple du « Pays des Étangs » en Moselle ", Territoire en mouvement, Revue de géographie et aménagement [En ligne], 22 | 2014, mis en ligne le 15 juin 2014, http://tem.revues.org/2415

Mathis D. et Mathis A., 2013 « Paysages et biodiversité hérités de l'exploitation du sel dans le Saulnois (Moselle) », Revue du Nord, Hors-série archéologie, $13^{\mathrm{e}}$ Rencontres internationales de Liessies, 174 p, p.145-154.

Mathis D. et Chiffre E., 2015, «Les grandes pêches : traditions renouvelées »,in Touchart L., Bartout P. et Motchalova O.,(dir.), Mieux comprendre les étangs. Expériences nationales et internationales, Brive, Éditions « les Monédières », 421 p., p.389-391.

Mathis D., Chiffre E., Harmand D., 2015, « Les enjeux paysagers de la reconversion en étangs des sablières et gravières ", in Touchart L., Bartout P. et Motchalova O., (dir.), Mieux comprendre les étangs. Expériences nationales et internationales, Brive, Éditions « les Monédières », 421 p., p.383-385. Papon P. et Touchart L. 2003 « Le Balaton, archétype du lac-étang ».Annales de Géographie, $\mathrm{n}^{\circ}$ 632, p.339-356.

Périgord M., Donadieu P., 2012, Le paysage, $2^{\mathrm{e}}$ édition, Armand Colin, 126 p.

Zapater M., Coquelet L., Clauce F., Poliautre P., Rousset X., 2010, « Le peuplier en zone humide », in Actes du colloque : Regards sur les tourbières des vallées alluviales, les peupleraies et le pâturage, p.158-168.

\section{NOTES}

1. $3^{\mathrm{e}}$ région aquacole de France.

2. Une mardelle est une "mare » dont l'origine est discutée. Il s'agit d'une petite "doline » marquée par des dépôts superficiels argileux imperméables créée par un phénomène karstique de dissolution des roches sous-jacentes (gypse, dolomie...). Les archéologues y voient la trace paysagère d'anciennes habitations, datant peut-être de l'âge du fer, creusées dans le sol établissant un « fond de cabane » aujourd'hui souvent rempli d'eau.

3. Abbaye cistercienne fondée par le duc de Lorraine Simon $1^{\mathrm{er}}$ en 1135 et destinée à devenir la nécropole ducale.

4. Le Grafenweiher a été détruit vers 1850 en raison de conflits liés à la ressource en eau. Le site est alors drainé, et transformé en prairie de fauche. Finalement avec la déprise une aulnaie est plantée en 1960 alors que les prairies sont délaissées.

5. La militarisation de la région est liée à l'affrontement direct entre les ducs de Lorraine et les comtes de Lichtenberg soutenus par les empereurs Hohenstaufen.

6. Abbaye cistercienne fondée vers 1133-1135.

7. Abbaye cistercienne fondée en 1128, confirmée en 1134 .

8. Queue ou cornée (Lorraine), espace amont correspondant à un ancien vallon souvent en tête de bassin, inondé lors de la mise en eau d'un étang. Sa forme triangulaire comparable à une corne 
explique cette dénomination. Les grands étangs, aux berges fortement découpées, se composent en plusieurs cornées.

9. Le domaine du Lindre (12 étangs) a été acheté par le département de la Moselle en 1975, après un montage complexe pour la somme de 15 millions de francs. La plaine et l'étang du Bischwald ont été acquis par la CCCMavec des participations de la Région Lorraine (20\%), du Conseil Général de la Moselle (20\%) et de l'Agence de l'Eau Rhin Meuse (60\%).

\section{ABSTRACTS}

Lorraine has an important ponds heritage for an agro-fishing activity. From 19th century, these systems have declined, weakened by emerging issues management and use of water resources. The ponds have then been abandoned as productive space. Nowadays, these water territories are inscribed in the heart of territorial projects. Its built on new uses combining environmental approaches and activities of leisure and tourism. However, constraints of protection and preservation and use of the terroirs of water generate conflicts of use while on the outskirts of urban areas are built by new terroirs of ponds, by the conversion of the aggregate extraction sites in valleys funds. These new converted ponds meet the demands of "waterscapes" urban populations.

La Lorraine dispose d'un important patrimoine d'étangs destiné à une activité agro-piscicole. À partir du XIX ${ }^{\mathrm{e}}$ siècle, ces systèmes ont décliné, fragilisés par les nouveaux enjeux de gestion et d'utilisation de la ressource en eau. Les étangs ont alors été délaissés comme espace productif. Aujourd'hui ces territoires d'eau sont inscrits au cœur de projets territoriaux construits sur de nouveaux usages associant démarches environnementales et activités de loisirs et de tourisme. Toutefois, les contraintes de protection et de préservation, l'utilisation des terroirs d'eau génèrent des conflits d'usage, alors qu'à la périphérie des aires urbaines se construisent de nouveaux terroirs d'étangs par la reconversion des sites d'extraction des granulats dans les fonds de vallées. Ces nouveaux étangs reconvertis répondent aux demandes de " paysages d'eau » des populations urbaines.

\section{INDEX}

Mots-clés: étangs, Lorraine, gravières, pisciculture, protection

Keywords: ponds, gravel pits, fish farming

Schlüsselwörter. Weiher, Lothringen, Baggersee, Fischzucht, Naturschutz

\section{AUTHORS}

\section{DENIS MATHIS}

Docteur en géographie, chercheur associé - Université de LorraineLaboratoire d'Observation des TERRitoires (LOTERR)23, boulevard Albert $1^{\mathrm{er}}$ - BP 33-97 - 54015 NANCY CEDEX Tél :

03.54.50.51.27 Mail : denis.mathis[chez]ac-nancy-metz.fr 


\section{ANNE MATHIS}

Master en géographie, Chercheuse associée - Université de LorraineLaboratoire d'Observation des TERRitoires (LOTERR)23, boulevard Albert 1 ${ }^{\mathrm{er}}$ - BP 33-97 - 54015 NANCY CEDEXTél :

03.54.50.51.27 Mail : Mathisanne[chez]aol.com 